\title{
DIET-DISEASE RELATIONSHIPS
}

\section{Recent advances in nutritional epidemiology}

RAUL ZAMORA-ROS AND CARLOS ALBERTO GONZÁLEZ

Nutritional epidemiology currently studies the diet-disease relationships. In order to evaluate these associations, an accurate estimation of nutritional exposure is essential. Traditional dietary questionnaires are being complemented with the measurement of nutritional biomarkers. New methodologies, including the use of new dietary assessments, metabolomics for increasing the quantity and quality of biomarkers, and statistical approaches to combine both techniques, are required to move forward in this field. In this review, we have selected five of the more relevant accomplishments in this field as examples of the importance of dietary factors in the prevention of non-communicable diseases. This theoretical knowledge needs to be finally translated by public health experts into dietary recommendations to the general population.

Keywords: nutritional epidemiology, fruits and vegetables, red and processed meat, sugarsweetened beverages, Mediterranean diet.

\section{INTRODUCTION}

Nutritional epidemiology is the study of human health in relation to nutrition. In the beginning, it was considered as a small subdiscipline of epidemiology, but since some decades ago it has grown into a branch with a major public health importance. Its historical role was to identify essential nutrients, in order to prevent nutritional deficiencies.

Nowadays, its purpose is to investigate the relationship between diet (i.e., dietary pattern, foods, and nutrients) and disease. Finally, public health nutrition converts this knowledge into practice, in order to use nutrition to prevent especially chronic diseases and increase life span and quality of life (Boeing, 2013).

\section{«Nutritional epidemiology is a scientific discipline that has grown a lot in the last decades and its role is essential to unravel the diet-disease relationship»}

common method in large epidemiological studies to assess the long-term dietary exposure, because they are simple, fast, and cheap; but they are less accurate and reliable than other methods, such as food records. Nevertheless, all self-reported dietary data are typically subject to random and systematic error during the dietary assessment process. Random errors (e.g., people can randomly under- or over-report) decrease the precision of the measurements, causing a loss in the statistical power. Whereas, systematic errors (e.g., a group of people, like people with obesity, may systematically underreport) reduce the accuracy of measurements generating bias in the relative risks. In combination, random variation
The main pillars of nutritional epidemiology are the estimation of dietary and nutritional exposure, and the statistical modeling of the diet-disease relationship (Boeing, 2013). In order to evaluate dietary exposure, traditional dietary assessment methods have been used, such as foodfrequency questionnaires (FFQ), 24-h dietary recalls (24-HDR), and food diaries. The FFQs are the most usually prevails, still leading to overall attenuation of the relative risk estimate. Recently, innovative technologies have been developed to improve especially self-reported assessments, such as the collection of multiple 24-HDRs and food records and interactive computer- and camera-based technologies (Illner et al., 2012). 
In order to evaluate nutritional exposure, a dietary assessment method and a food composition table/ database are required. Random and systematic errors are also probable in food composition data. True variability of the nutrient content in foods causes random errors; while biased food composition data due to, for example the use of an incorrect analytical method, causes systematic error. Substantial advances in this field to avoid these issues has been accomplished in the last years, having more comprehensive current food composition databases, with an increasing number of foods (e.g., new products, less habitual foods, different varieties, different cooking methods), with a higher amount of nutrients and compounds (e.g., amino acids, individual fatty acids, polyphenols, contaminants, etc.), and including data from specific countries and regions (e.g., databases from African, Latin American, or Asian countries).

Nutritional biomarkers are an alternative method to assess dietary intake. They present several advantages compared to dietary questionnaires: they are generally more accurate, objective, and also take into account bioavailability (Potischman, 2003). Although traditional approaches continue to provide valuable knowledge about the cause of chronic diseases, new technologies will be essential to maximize the impact of nutritional epidemiology in the future. Nowadays, metabolomics is becoming the technology of reference to evaluate multiple biomarkers simultaneously (Ulaszewska et al., 2018). For example, a recent study has discovered a metabolic fingerprint of the adherence to the Mediterranean diet, based on 67 metabolites using targeted metabolomics. Moreover, it was associated with a lower cardiovascular disease risk in the PREDIMED study and the US Nurses' Health Studies I and II, and Health Professionals Follow-up Study (Li et al., 2020).

Advances in statistical modeling (calibration and mediation analysis) are also basic to integrate and exhaustively explore the relationships between dietary factors, biomarkers, and health status (BrandoliniBunlon et al., 2019; Shaw et al., 2018). Furthermore, new statistical approaches are needed to combine data from dietary data and nutritional biomarkers.
Currently, two predominant methods, the calibration and the triads methods have been proposed (Gormley et al., 2020). The Howe's method has been also used. It is a simple technique, in which participants are ranked from the lowest to the highest value for reported dietary intake and biomarker level, and then the two scores are summed obtaining a continuous score that can be converted into quantiles (Freedman et al., 2010).

In this manuscript, we present five essential studies that have enormously contributed with the current knowledge on nutritional epidemiology as examples of the impact and advances on this field in the last lustrum.

\section{GLOBAL HEALTH EFFECTS OF DIETARY RISKS}

The Global Burden of Disease Study 2017 (GBD 2017 Diet Collaborators, 2019) is the widest and most recent published study aimed to evaluate the consumption of fifteen main foods and nutrients across 195 countries worldwide and to quantify the 


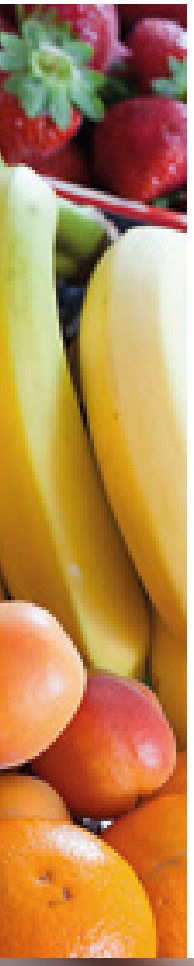

impact of their intake on non-communicable diseases. The study reports data from multiple sources on intake, collected between 1990 and 2016 among adults. Regarding the associations with diseases, data from published meta-analysis of prospective observational studies were used to estimate mortality and morbidity risks.

Globally, in 2017, dietary factors were responsible for 11 million deaths, which represent the $22 \%$ of all deaths among adults, and 255 million disabilityadjusted life-years (DALYs) ${ }^{1}$, which represent $15 \%$ of all DALYs among adults. Cardiovascular disease was the leading cause of diet-related deaths (10 million deaths) and DALYs (207 million) followed by cancer (913,090 deaths and 20 million DALYs), and type 2 diabetes (T2D) (338,714 deaths and 24 million DALYs). High intake of sodium (3 million deaths and 70 million DALYs), low intake of whole

\footnotetext{
${ }^{1}$ An indicator that quantifies the years of healthy and full life that are potentially lost due to the disabling consequences of illness.
}

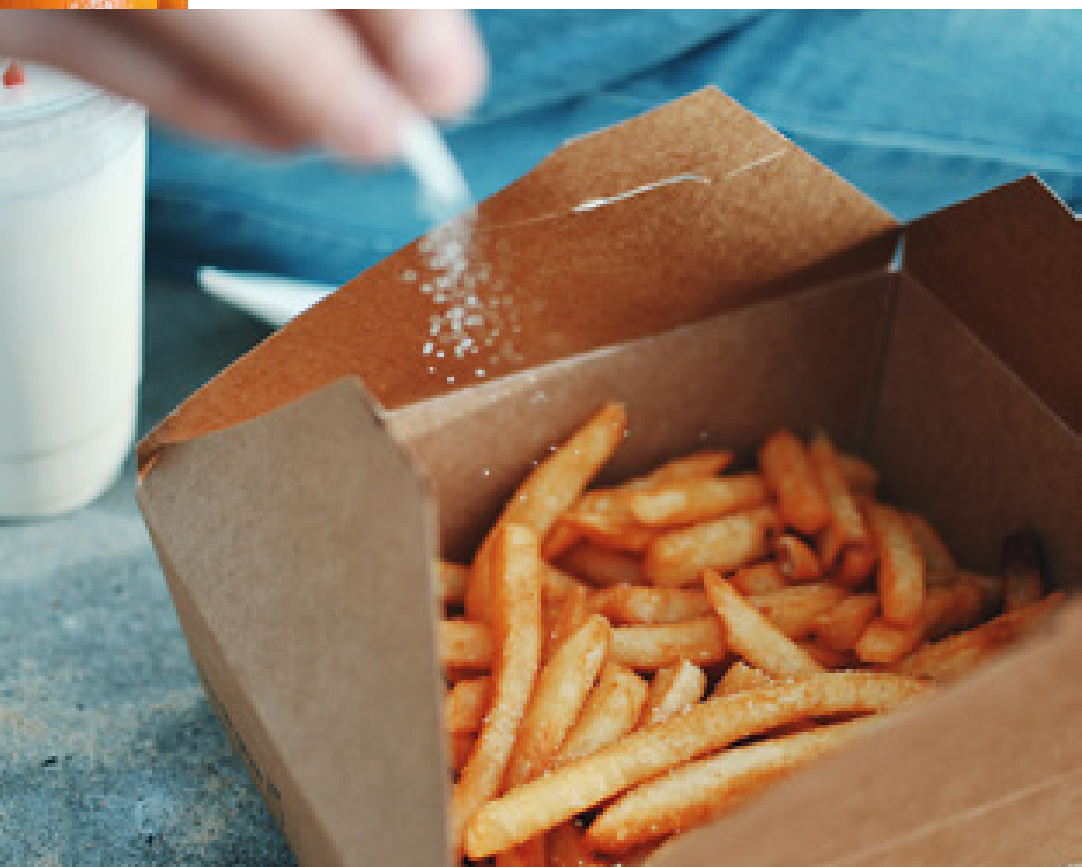

High intake of sodium, low intake of whole grains, and low intake of fruits were the leading dietary risk factors for deaths and disability-adjusted life-years globally in many countries.

\section{«Globally, in 2017, dietary factors were responsible for 11 million deaths, which represent the $22 \%$ of all deaths among adults»}

grains ( 3 million deaths and 82 million DALYs), and low intake of fruits (2 million deaths and 65 million DALYs) were the leading dietary risk factors for deaths and DALYs globally in many countries. More than half of all diet-related deaths and two-thirds of diet-related DALYs were attributable to just these three dietary factors and there was a disproportionate burden in low-income settings. They concluded claiming that the finding highlights the urgent need for coordinated global efforts to improve the quality of human diet.

Regarding the need for improving diet across nations, it is important to take into account the comment by Drs. Nita Forouhi and Nigel Unwin (2019, p. 1918) that go with their original article in The Lancet: «There are of course considerable challenges in shifting populations' diets in this direction, illustrated by the cost of fruits and vegetables being disproportionately prohibitive: two servings of fruits and three servings of vegetables per day per individual accounted for $52 \%$ of household income in low-income countries, $18 \%$ in low to middle income countries, $16 \%$ in middle to upper income countries, and $2 \%$ in high-income countries.» Therefore, without an integrated policy intervention not only to improve the availability of healthy foods, but also to reduce the social inequalities and to improve the welfare state in the world will be difficult to reduce the burden of non-communicable diseases associated to diet.

\section{FRUIT AND VEGETABLE INTAKE AND THE RISK OF MAJOR CHRONIC DISEASES}

High consumption of fruit and vegetables is one of the most usual recommendations for a healthy diet to reduce the risk of cancer and cardiovascular disease (CVD), which are the two most common chronic diseases worldwide. These recommendations are based on hundreds of epidemiological studies, although the strength and shape of the dose-response relationships between fruit and vegetables intake and the risk of these diseases is still uncertain.

A systematic review and meta-analysis was conducted (Aune et al., 2017), including 95 prospective studies (142 publications) published up to September 2016, from various geographical localization, except Africa, West Asia, South and Latin America, due to the absence of such studies in these areas. Globally, there were analyzed 43,000 
coronary heart disease cases, 47,000 stroke cases, 81,000 cardiovascular disease cases, 112,000 cancer cases, and 94,000 deaths among up to 2.1 million participants.

For fruit and vegetables combined, the summary relative risks (RRs) per $200 \mathrm{~g} /$ day was $0.92(95 \%$ confidence interval (CI) 0.90-0.94) for coronary heart disease, 0.84 (95\% CI, 0.76-0.92) for stroke, 0.90 (95\% CI, 0.90-0.95) for CVD, 0.97 (95\% CI, 0.95-0.99) for total cancer, and 0.90 (95\% CI, 0.87-0.93) for all-cause mortality. Similar results were observed for fruits and vegetables separately. When dose-response relationships were analyzed, evidence of nonlinearity was detected in all analyses. For fruits and vegetables combined the lowest risk was observed at intake doses of 550-600 g/day (7-7.5 serving/day) for total cancer, with little evidence of further risk reductions at higher intakes. For coronary disease, stroke, CVD, and all-cause mortality the lowest risk was observed at $800 \mathrm{~g} /$ day (10 serving/ day). An estimated of 7.8 million premature deaths worldwide in 2013 may be attributable to a fruit and vegetables intake below $800 \mathrm{~g} /$ day, if the observed associations are causal. In conclusion, this study supports public health recommendations to increase fruit and vegetables intake for the prevention of CVD (>800 g/day) and cancer (>600 g/day).

\section{DISEASES RELATED TO SUGAR- SWEETENED BEVERAGE CONSUMPTION}

Sugar-sweetened beverages (SSBs) are the single largest source of added sugar and a top source of energy intake in the U.S. diet, as well as in most countries worldwide. A recent published systematic review of randomized control trials and prospective studies have provided sufficient evidence ( $\mathrm{Hu}, 2013)$ to conclude that high intake of SSBs is causally associated with obesity in adults, children, and adolescents, and to demonstrate a direct dose-response relationship between SSBs consumption and long-term weight gain and risk of T2D. Trend analyses in adult body mass index (BMI) in 200 countries, from 1975 to 2014 (NCD Risk Factor Collaboration, 2016), have shown the current epidemic burden of obesity globally, which has become one of the main public health concerns worldwide.

This study (Singh et al., 2015) showed the impact of SSBs consumption on burdens of adiposity-related
CVD, cancers, and T2D, by nation, age, and sex in 2010. Data on SSB consumption were pooled from national dietary surveys worldwide. Disease-specific mortality/ morbidity data were obtained from the Global Burden of Disease 2010 Study. According to the study, in 2010, the mean global consumption of SBBs among adults was 0.58 servings/day. Furthermore, in 2010 , a total of 184,000 deaths globally - representing $5.3 \%$ of all diabetes death, $0.4 \%$ of BMI-related CVD deaths, and $0.3 \%$ of all BMI-related cancer deaths - were attributed to SSB consumption. A $72.3 \%$ of these deaths $(n=133,000)$ were due to T2D, $24.2 \%(n=45,000)$ to CVD, and $3.5 \%$ $(n=6,450)$ to BMI-related cancers. A total of 8.5 million DALYs were related to SBBs intake. About $75 \%$ of all deaths attributable to SSB consumption occurred in low-and middle-income countries and $24 \%$ in high-

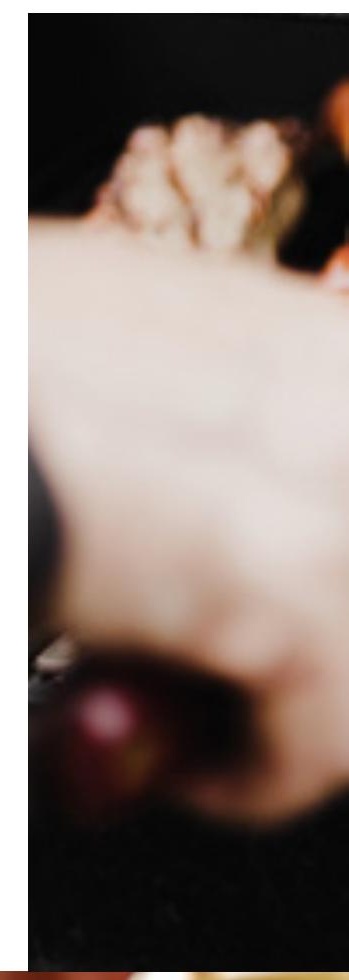

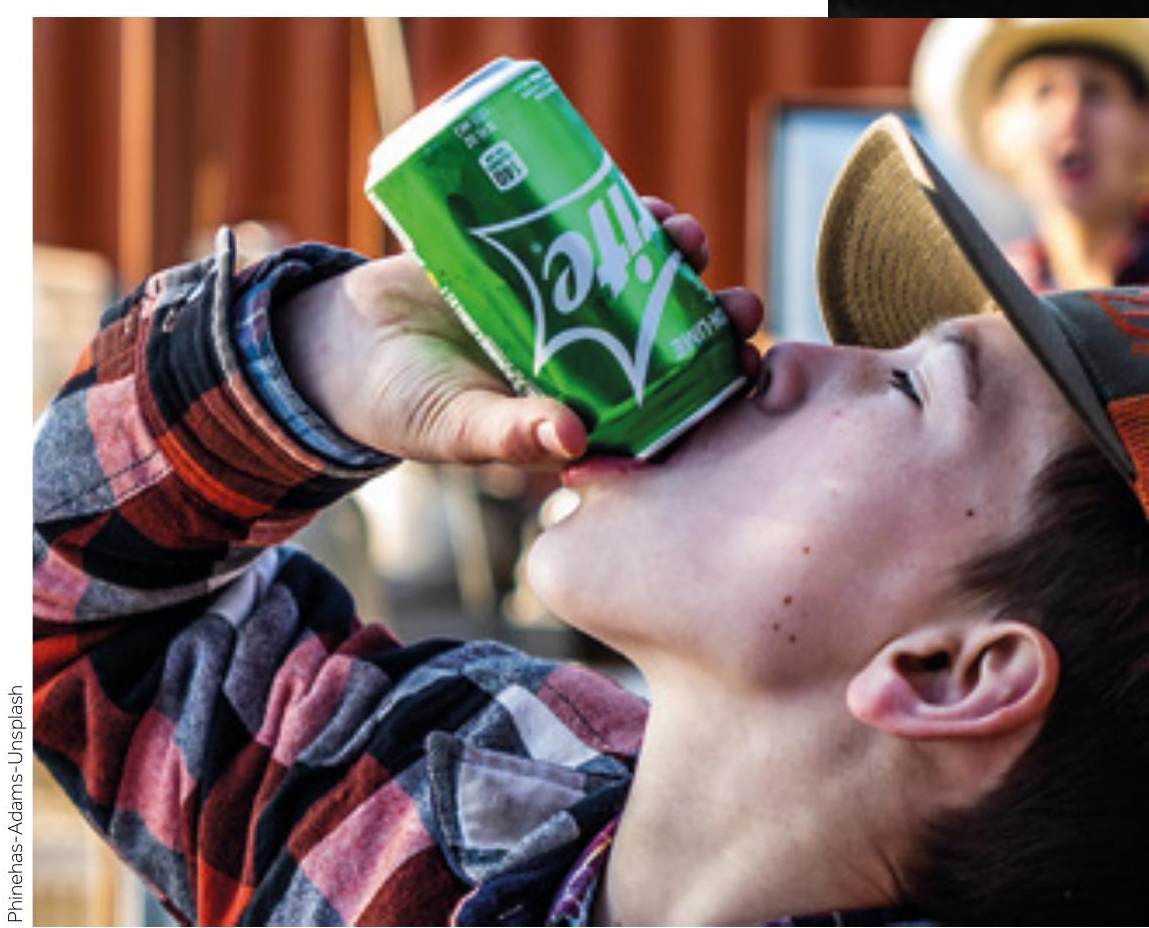

Sugar-sweetened beverages (SSBs) are the single largest source of added sugar and a top source of energy intake in the U.S. diet, as well as in most countries worldwide.

\section{«A high intake of sugar-sweetened beverages is causally associated with obesity in adults, children and adolescents»}


of the evidence, the results of the meta-analyses conducted by both groups of experts are identical: red and processed meats are associated with a higher risk of colorectal cancer. Therefore, in our opinion, dietary guidelines should not be changed and the precautionary principle in public health should be applied until the opposite is demonstrated. Thus, the recommendation should be to limit the consumption of red meat and processed meat to no more than three and one portions per week, respectively; in order to improve both human health and environmental sustainability.

\section{- PRIMARY PREVENTION OF CARDIOVASCULAR DISEASE WITH A MEDITERRANEAN DIET}

The traditional Mediterranean diet (MD) is characterized by a high intake of fruit, vegetables, nuts, legumes, olive oil, and cereals; a moderate intake of fish, eggs, and poultry; a low intake of dairy products, red and processed meats, and sweets; and wine in moderation, consumed mainly with meals. Over the last few decades, it has been promoted worldwide as one of the healthiest dietary patterns and has been reported to be consistently beneficial with respect to chronic diseases and longevity (Galbete et al., 2018). However, most of these findings were concluded from observational studies, so a large clinical trial on traditional MD on hard endpoints was missing.

The PREDIMED (Prevention with Mediterranean Diet) study is a parallel, multicenter clinical trial conducted in Spain (Estruch et al., 2018). Due to some inconsistences in the randomization of the subjects, the study is not completely randomized. For this reason, the study was retracted and reanalyzed from the previous version in 2013. The study included almost 7,500 participants (55 to 80 years of age, $57 \%$ women) who were at high cardiovascular risk, but with no CVD at enrollment, and were «practically» randomly assigned to one of three diets: a MD supplemented with extra-virgin olive oil, a MD supplemented with mixed nuts, or a control
The traditional Mediterranean diet is characterized by a high intake of fruit, vegetables, nuts, legumes, olive oil, and cereals; a moderate intake of fish, eggs and poultry; a low intake of dairy products, red and processed meats, and sweets; and wine in moderation, consumed mainly with meals.

\section{«Over the last few decades, Mediterranean diets have been reported to be consistently beneficial with respect to chronic diseases and longevity»}

diet (advice to reduce dietary fat). After 4.8 years of median follow-up, 288 participants had a primary end-point (i.e., myocardial infarction, stroke, and death from cardiovascular causes). In the intention-totreat analysis including all the participants and adjusting for potential confounders, participants following a MD with extra-virgin olive oil reduced the risk of primary end-points in $31 \%$ (95\% CI, 0.53-0.91) compared to those with the control diet. Whereas, individuals in the group of the MD with nuts had a reduced $28 \%$ risk (95\% CI, 0.54-0.95) compared to those with the control diet.

This was the first interventional and large study showing the advantages of following a healthy diet, in this case the traditional MD, in the primary prevention of cardiovascular disease. This study also provided valuable evidence regarding the beneficial effects of MD against other chronic diseases, such as obesity, type- 2 diabetes, and breast cancer. 


\section{CONCLUSIONS}

Nutritional epidemiology is a scientific discipline that has grown a lot in the last decades and its role is essential to unravel the diet-disease relationship. Here, we have presented five successful examples on the importance of dietary factors in the prevention of non-communicable diseases. Currently, this knowledge has being translated to dietary guidelines and prevention programs in order to provide dietary recommendations to the general population. $\odot$

\section{REFERENCES}

Aune, D., Giovannucci, E., Boffetta, P., Fadnes, L. T., Keum, N. N., Norat, T., Greenwood, D. C., Riboli, E., Vatten, L. J., \& Tonstad, S. (2017). Fruit and vegetable intake and the risk of cardiovascular disease, total cancer and all-cause mortality-a systematic review and dose-response meta-analysis of prospective studies. International Journal of Epidemiology, 46(3), 1029-1056. https://doi. org/10.1093/ije/dyw319

Boeing, H. (2013). Nutritional epidemiology: New perspectives for understanding the diet-disease relationship? European Journal of Clinical Nutrition, 67(5), 424-429. https://doi.org/10.1038/ ejen.2013.47

Bouvard, V., Loomis, D., Guyton, K. Z., Grosse, Y., Ghissassi, F. E., Benbrahim-Tallaa, L., Guha, N., Mattock, H., \& Straif, K. (2015). Carcinogenicity of consumption of red and processed meat. Lancet Oncology, 16(16), 1599-1600. https://doi.org/10.1016/S1470. 2045 (15) 00444-1

Brandolini-Bunlon, M., Pétéra, M., Gaudreau, P., Comte, B., Bougeard, S., $\&$ Pujos-Guillot, E. (2019). Multi-block PLS discriminant analysis for the joint analysis of metabolomic and epidemiological data. Metabolomics, 15(10), 134. https://doi.org/10.1007/s11306-019-1598-y

Estruch, R., Ros, E., Salas-Salvadó, J., Covas, M.-I., Corella, D., Arós, F., Gómez-Gracia, E., Ruiz-Gutiérrez, V., Fiol, M., Lapetra, J., LamuelaRaventos, R. M., Serra-Majem, L., Pintó, X., Basora, J., Muñoz, M. A., Sorlí, J. V., Martínez, J. A., Fitó, M., Gea, A., ... Martínez-González, M. A. (2018). Primary prevention of cardiovascular disease with a mediterranean diet supplemented with extra-virgin olive oil or nuts. New England Journal of Medicine, 378(25), e34. https://doi.org/10.1056/ NEJMoa1800389

Forouhi, N. G., \& Unwin, N. (2019). Global diet and health: Old questions, fresh evidence, and new horizons. Lancet, 393(10184), 1916-1918. https://doi.org/10.1016/S0140-6736(19)30500-8

Freedman, L. S., Tasevska, N., Kipnis, V., Schatzkin, A., Mares, J., Tinker, L., \& Potischman, N. (2010). Gains in statistical power from using a dietary biomarker in combination with self-reported intake to strengthen the analysis of a diet-disease association: An example from CAREDS. American Journal of Epidemiology, 172(7), 836-842. https://doi.. org/10.1093/aje/kwq194

Galbete, C., Schwingshackl, L., Schwedhelm, C., Boeing, H., \& Schulze, M. B. (2018). Evaluating mediterranean diet and risk of chronic disease in cohort studies: An umbrella review of meta-analyses. European Journal of Epidemiology, 33(10), 909-931. https://doi_org/10.1007/s10654018-0427-3

GBD 2017 Diet Collaborators. (2019). Health effects of dietary risks in 195 countries, 1990-2017: A systematic analysis for the Global Burden of Disease Study 2017. The Lancet, 393(10184), 1958-1972. https://doi. org/10.1016/S0140-6736(19)30041-8

Gormley, I. C., Bai, Y., \& Brennan, L. (2020). Combining biomarker and self-reported dietary intake data: A review of the state of the art and an exposition of concepts. Statistical Methods in Medical Research, 29(2), 617-635. https://doi.org/10.1177/0962280219837698

Hu, F. B. (2013). Resolved: There is sufficient scientific evidence that decreasing sugar-sweetened beverage consumption will reduce the prevalence of obesity and obesity-related diseases. Obesity Reviews, 14(8), 606-619. https://doi.org/10.1111/obr:12040
Illner, A.-K., Freisling, H., Boeing, H., Huybrechts, I., Crispim, S., \& Slimani, N. (2012). Review and evaluation of innovative technologies for measuring diet in nutritional epidemiology. International Journal of Epidemiology, 41, 1187-1203. https://doi.org/10.1093/ije/dys 105 Johnston, B. C., Zeraatkar, D., Han, M. A., Vernooij, R. W. M., Valli, C., El Dib, R., Marshall, C., Stover, P. J., Fairweather-Taitt, S., Wójcik, G., Bhatia, F., de Souza, R., Brotons, C., Meerpohl, J. J., Patel, C. J., Djulbegovic, B., Alonso-Coello, P., Bala, M. M., \& Guyatt, G. H. (2019). Unprocessed red meat and processed meat consumption: Dietary guideline recommendations from the nutritional recommendations (NutriRECS) Consortium. Annals Internal of Medicine, 171(10), 756-764. https://doi. org/10.7326/M19-1621

Li, J., Guasch-Ferré, M., Chung, W., Ruiz-Canela, M., Toledo, E., Corella, D., Bhupathiraju, S. N., Tobias, D. K., Tabung, F. K., Hu, J., Zhao, T., Turman, C., Feng, Y.-C. A., Clish, C. B., Mucci, L., Eliassen, A. H., Costenbader, K. H., Karlson, E. W., Wolpin, B. M., ... Liang, L. (2020). The mediterranean diet, plasma metabolome, and cardiovascular disease risk. European Heart Journal, ehaa209. https://doi.org/10.1093/ eurheartj/ehaa209

NCD Risk Factor Collaboration. (NCD-RisC). (2016). Trends in adult bodymass index in 200 countries from 1975 to 2014: A pooled analysis of 1698 population-based measurement studies with $19 \cdot 2$ million participants. The Lancet, 387(10026), 1377-1396. https://doi.org/10.1016/S0140$6736(16) 30054-\mathrm{X}$

Potischman, N. (2003). Biologic and methodologic issues for nutritional biomarkers. Journal of Nutrition, 133(3), 875S-880S. https://doi. org/10.1093/jn/133.3.875S

Shaw, P. A., Deffner, V., Keogh, R. H., Tooze, J. A., Dodd, K. W. Küchenhoff, H., Kipnis, V., Freedman, L. S., Measurement Error and Misclassification Topic Group (TG4) of the STRATOS Initiative. (2018). Epidemiologic analyses with error-prone exposures: Review of current practice and recommendations. Annals of Epidemiology, 28(11), 821-828. https://doi.org/10.1016/j.annepidem.2018.09.001

Singh, G. M., Micha, R., Khatibzadeh, S., Lim, S., Ezzati, M., Mozaffarian, D., \& Global Burden of Diseases Nutrition and Chronic Diseases Expert Group (NutriCoDE). (2015). Estimated global, regional, and national disease burdens related to sugar-sweetened beverage consumption in 2010. Circulation, 132(8), 639-666. http://doi.org/10.1161/ CIRCULATIONAHA.114.010636

Ulaszewska, M. M., Weinert, C. H., Trimigno, A., Portmann, R., Andres Lacueva, C., Badertscher, R., Brennan, L., Brunius, C., Bub, A., Capozzi, F., Cialiè Rosso, M., Cordero, C. E., Daniel, H., Durand, S., Egert, B., Ferrario, P. G., Feskens, E. J. M., Franceschi, P., Garcia-Aloy, M., .. Vergères, G. (2018). Nutrimetabolomics: An integrative action for metabolomic analyses in human nutritional studies. Molecular Nutrition \& Food Research, 63(1), e1800384. https://doi.org/10.1002/ mnfr. 201800384

Vieira, A. R., Abar, L., Chan, D. S. M., Vingeliene, S., Polemiti, E., Stevens, C., Greenwood, D., \& Norat, T. (2017). Foods and beverages and colorectal cancer risk: A systematic review and meta-analysis of cohort studies, an update of the evidence of the WCRF-AICR Continuous Update Project. Annals of Oncology, 28(8), 1788-1802. https://doi. org/10.1093/annonc/mdx171

ACKNOWLEDGEMENTS: RZ-R has been supported by the Miguel Servet program (CP15/00100) from the Institute of Health Carlos III (Co-funded by the European Social Fund (ESF) - ESF investing in your future).

RAUL ZAMORA-ROS. Miguel Servet Researcher in the Unit of Nutrition and Cancer, Cancer Epidemiology Research Program, Catalan Institute of Oncology, Bellvitge Biomedical Research Institute (IDIBELL), L'Hospitalet de Llobregat, Barcelona (Spain). His research focuses on investigating whether dietary factors, particularly polyphenols and polyphenol-rich foods, are causally associated with the development of chronic diseases. $\triangle$ rzamora@idibell.cat

CARLOS ALBERTO GONZÁLEZ. He is an emeritus researcher and former Head of the Unit of Nutrition and Cancer, Cancer Epidemiology Research Program, Catalan Institute of Oncology, Bellvitge Biomedical Research Institute (IDIBELL), L'Hospitalet de Llobregat, Barcelona (Spain). His research focuses on the field of nutritional epidemiology, particularly in dietcancer relationships. $\square$ cagonzalez@iconcologia.net 